\title{
Data mining and association rules to determine twitter trends
}

\author{
Autores \\ Jesús Silva, Jesús Vargas, Domingo Natteri, Darío Flores, Omar Bonerge Pineda Lezama, \\ Bridy Ahumada, Lesbia Valero
}

\begin{abstract}
Opinion mining has been widely studied in the last decade due to its great interest in the field of research and countless real-world applications. This research proposes a system that combines association rules, generalization of rules, and sentiment analysis to catalog and discover opinion trends in Twitter [1] ]. The sentiment analysis is used to favor the generalization of the association rules. In this sense, an initial set of 1.6 million tweets captured in an undirected way is first summarized through text mining in an input set for the algorithms of rules and sentiment analysis of 158,354 tweets. On this last group, easily interpretable standard and generalized sets of rules are obtained about characters, which were revealed as an interesting result of the system.

Palabras clave
\end{abstract}

Opinions mining, Association rules, Sentiment analysis, Analysis of trends, Unsupervised learning. 